\title{
Técnica modificada de postioplastia en toros cebuínos con balanopostitis ulcerativa del trópico mexicano
}

Modified postioplasty technique in cebuíno bulls with ulcerative balanoposthitis of the Mexican tropics

Arieta R.R.J. ${ }^{1}$, Bailón B.A. ${ }^{2}$, Graillet J.M,E, ${ }^{1}$., Fernández F.J.A. ${ }^{1}$, Alvarado G.L.C. ${ }^{1}$, Martínez M.M. ${ }^{1}$

${ }^{1}$ Facultad de Ingeniería en Sistemas de Producción Agropecuaria. Universidad Veracruzana Carretera Costera del Golfo km 220, Col. Agrícola Micahapan C.P. 96100. ${ }^{2}$ Facultad de Medicina Veterinaria y Zootecnia UNAM - Av. Universidad No. 3000 Colonia UNAM, C.U., Del. Coyoacán, 04510.

Recibido: 05/08/2019

Aceptado: 13/12/2019

\section{RESUMEN}

El objetivo del presente trabajo es mostrar los resultados de toros con postitis que se les practicó Técnica Modificada de Postioplastia como manejo quirúrgico de esta patología. Se realizó un estudio de tipo descriptivo no probabilístico en toros reproductivamente activos que presentaron postitis crónica ulcerativa. Los casos clínico-quirúrgicos se presentaron en diferentes ranchos de la zona sur de Veracruz. Los pacientes fueron 10 toros cebús de los cuales 7 eran de la raza brahmán, 1 indubrasil y 2 Sardo Negro, con buena condición corporal y con postitis crónica ulcerativa. Ninguno de los animales había recibido tratamiento quirúrgico. Se aplicó xilacina al $2 \%$ a la dosis de $0,1 \mathrm{mg} / \mathrm{kg}$ de peso vivo como tranquilizante por vía intramuscular. Posteriormente, para la anestesia, se infiltró clorhidrato de lidocaína al $2 \%$ a dosis de $1 \mathrm{ml} / \mathrm{cm}^{3}$ de tejido en la base del prepucio. Una vez tranquilizado el paciente y realizadas las maniobras de derribo, se hizo lavado y evaluación detallada de la lesión. Se realizó una incisión en el limbo mucocutáneo y no en piel para reducir las posibilidades de fimosis cicatrizal. Se ligaron los vasos de gran calibre con puntos de transfixión con material absorbible acidó poliglicólico del calibre 0. Para la hemostasia de los vasos pequeños fue suficiente el pinzamiento y torsión. La divulsión se realiza en $360^{\circ}$ hasta conseguir el desprendimiento de todo el tejido afectado incluyendo la mucosa prepucial dañada. Una vez practicada la correcta hermostasis se procedió sujetar la mucosa con pinzas de goma para evitar la retracción, así mismo se le colocaron 4 puntos de forma cardinal con sutura de nylon monofilico para expandir la mucosa y poder suturarla a la piel, esos mismo puntos de sujeción se fijaron con punto de cirujano y posteriormente se colocaran 4 puntos entre ellos. Los 10 toros operados con la técnica descrita tuvieron recuperación en un período de 90 días posoperatorio en promedio, dos de ellos tuvieron complicación de estenosis cicatrizal. Se concluye que la técnica quirúrgica empleada en este estudio tuvo resultados favorables. Se considera que si el posoperatorio no se lleva correctamente trasciende seriamente en el éxito o fracaso de esta cirugía.

Palabras clave: Postioplastia, postitis. 


\begin{abstract}
The objective of the present work is to show the results of bulls with postitis that were Modified Postioplasty Technique as surgical management of this pathology. A non-probabilistic descriptive study was conducted in reproductively active bulls that presented chronic ulcerative postitis. Clinical-surgical cases occurred in different ranches in the southern area of Veracruz. The patients were 10 bulls bulls of which 7 were of the Brahmin breed, 1 indubrasil and 2 Black Sardo, with good body condition and with chronic ulcerative postitis. None of the animals had received surgical treatment. $2 \%$ xylazine was applied at the dose of $0.1 \mathrm{mg} / \mathrm{kg}$ body weight as a tranquilizer intramuscularly. Subsequently, for anesthesia, $2 \%$ lidocaine hydrochloride was infiltrated at a dose of $1 \mathrm{ml} / \mathrm{cm} 3$ of tissue at the base of the foreskin. Once the patient was reassured and the demolition maneuvers were performed, washing and detailed evaluation of the lesion was done. An incision was made in the mucocutaneous limbus and not in the skin to reduce the chances of scar phimosis. Large caliber vessels were ligated with transfixion points with 0 . caliber polyglycolic acid absorbable material. For clamping and small vessel hemostasis, tightening and torsion was sufficient. The dissemination is carried out in $360^{\circ}$ until the detachment of all the affected tissue including the damaged prepucial mucosa is achieved. Once the correct beauty was performed, the mucosa was clamped with rubber tweezers to prevent retraction, and 4 cardinal points with monophilic nylon suture were placed to expand the mucosa and be able to suture it to the skin, those same points of clamping were fixed with surgeon point and subsequently 4 points were placed between them. The 10 bulls operated with the technique described had recovery in a period of 90 days postoperatively on average, two of them had complication of scar stenosis. It is concluded that the surgical technique used in this study had favorable results. It is considered that if the postoperative is not carried correctly it transcends seriously in the success or failure of this surgery.
\end{abstract}

Keywords: Postioplasty, postitis.

\section{INTRODUCCIÓN}

En los países tropicales se presenta una incidencia alta en problemas prepuciales en toros cebú, debido a la conformación misma del prepucio, a las particularidades de dicho tubo que al quedar prolapsado después de la monta queda expuesto a traumas; y al tipo de pastos que cortan el tubo estando prolapsado (1). El término postitis o acrobustitis se utiliza para describir la afección de la porción distal del aparato reproductor del macho bovino. Es un proceso inflamatorio crónico, en el cual se presenta el prolapso de la mucosa prepucial por traumatismo $\mathrm{y}$, por consiguiente, el estrechamiento del canal y la no exteriorización del pene (fimosis) $(7,2)$. Dentro de las casusas que predisponen a la patología en el Bos taurus indicus están las morfohereditarias (poco desarrollo o ausencia de músculo prepucial, escaso desarrollo del músculo retractor del pene, orificio prepucial amplio con capacidad limitada para contraerse, vaina colgante y larga), que hacen al prepucio susceptible a la exposición ambiental y al consiguiente daño por trauma (4). Le siguen las traumáticas, que generan heridas y cicatrices en mucosa (pasturas infestadas de maleza y plantas espinosas, cercas de alambres de púa, el pisoteo autoinfringido y por otros animales), infecciosas pruriginosas (IBR, vibriones, tricomonas, estreptococos $\mathrm{y}$ estafilococos, variedades de la E. coli y 
actinomyces) (8-4). La postitis siempre se asocia con la balanitis, que es causada por infecciones bacterianas primarias con desvitalización de la vaina prepucial.

Factores secundarios asociados a postitis son la fractura de pene y la consiguiente acumulación de la orina, seguidos por un absceso prepucial. Estos trastornos son comunes en el prepucio de los toros, y se producen con mayor frecuencia en la época de reproducción (7). El diagnóstico se realiza a través de la observación de los cambios clínicos y de comportamiento del animal, como la dificultad o imposibilidad para lograr la cópula, los diversos grados de edema, la necrosis de la mucosa prolapsada, las larvas de mosca, la hemorragia, el absceso y la hipertermia local (8). El pene no puede ser expulsado de la cavidad prepucial o muestra dolor al retraerlo (parafimosis); el animal en algunos casos puede mostrar signos de estranguria y disuria. La estenosis de la abertura prepucial, la desviación del prepucio en dirección a su base y una orina acumulada en la cavidad interna de la vaina desencadenan una reacción local inflamatoria intensa, caracterizada por celulitis, que conduce a daños en la mucosa, tejido conectivo y hasta formaciones de absceso complicante, lo cual hace al toro inviable para la reproducción (7). Según Siqueira, Bernis y Bernis (9), la forma de describir el proceso es la siguiente: el proceso inicia como una inflamación aguda de la mucosa prepucial, caracterizada por edema y ganancia de peso que dificultan su reintroducción e incrementan su deshidratación y resequedad, la formación de úlceras que van desde únicas hasta múltiples. Estas úlceras pueden ser profundas (afectar la submucosa); de esta forma inician la lesión del tejido conectivo, que en su respuesta cicatrizal comienza la síntesis de colágeno y tejido fibroso con el propósito de reparar y delimitar el proceso. Sin embargo, en este proceso crónico mucosal la tendencia es a aumentar por el trauma reiterativo, resequedad, contaminación bacteriana, descomposición del esmegma y estrechez del ostio, que reducen las posibilidades de salida del pene, lo cual obliga a que el animal se orine dentro del prepucio, con salida a gotas de la orina y retención de otra gran parte que se descompone en sus derivados amoniacales. Esto causa irritación y necrosis de la mucosa y hasta del tejido subcutáneo con la consecuente formación de bridas fibrosas $o$ anillos, y hasta complicaciones bacterianas que terminan en flemones y abscesos que incluso pueden afectar al pene con inflamación de su mucosa y aun necrosis (los productos de desecho son eliminados por fagocitosis). El tratamiento farmacológico e higiénico se basa en la aplicación de antibióticos, desinflamatorios sistémicos y tópicos, lavados del prepucio y el uso de duchas frías. La colocación de vendas, ataduras y cintas adhesivas alrededor de la extremidad prepucial y la introducción de un tubo de polivinilo en el lumen prepucial facilitan el paso de la orina y previenen la estenosis, lo que ayudaría en el tratamiento del edema. Se recomienda reposo sexual para facilitar el proceso y prevenir la propagación de infecciones $(3,10,12)$. El tratamiento quirúrgico de la enfermedad está destinado a pacientes con daños en la mucosa o tegumento, para lo cual se utilizan varias técnicas como la postiectomía (6), la circuncisión prepucial en forma de v (10) y la utilización de anillos plásticos $(10,12)$. Cada paciente debe estudiarse detenidamente para establecer la recomendación más adecuada a su situación.De los tres procedimientos utilizados en la terapia quirúrgica de la postitis, tal vez la más aceptada es la postiectomía. En este sentido, el objetivo del presente trabajo es mostrar los resultados con la técnica modificada de postioplastía en toros cebuínos con balanoposstitis ulcerativa del trópico mexicano. 


\section{MATERIALES Y MÉTODOS}

\section{Tipo de estudio}

Se realizó un estudio de tipo descriptivo no probabilístico en toros reproductivamente activos que presentaron postitis crónica ulcerativa.

\section{Localización}

Los casos clínico-quirúrgicos se presentaron en diferentes ranchos de la zona sur de Veracruz. El estado está localizado en la parte oriental del país y es el décimo en la República Mexicana por su superficie: 72,410 kilómetros cuadrados, dividida en 203 municipios. De sur a norte el territorio veracruzano está comprendido por las coordenadas geográficas de $17^{\circ} 03^{\prime}$ y $22^{\circ} 27^{\prime}$ de latitud norte, y de este a oeste, entre los $93^{\circ} 36^{\prime}$ y $98^{\circ} 36^{\prime}$ de longitud oeste.

\section{Ejemplares}

Los pacientes fueron 10 toros cebús de los cuales 7 eran de la raza brahmán, 1 indubrasil y 2 sardo negro, con buena condición corporal y con postitis crónica ulcerativa. Ninguno de los animales había recibido tratamiento quirúrgico.

\section{Preanestesia y anestesia}

Se aplicó xilacina al $2 \%$ a la dosis de $0,1 \mathrm{mg} / \mathrm{kg}$ de peso vivo como tranquilizante por vía intramuscular. Posteriormente, para la anestesia, se infiltró clorhidrato de lidocaína al $2 \%$ a dosis de $1 \mathrm{ml} / \mathrm{cm}^{3}$ de tejido en la base del prepucio.

Cabe señalar que la contención de cada paciente fue practicada en decúbito lateral derecho con la debida protección de miembros torácicos y pélvicos, cabeza y región escapular, y que previo a la anestesia local se rasuró el campo operatorio, se lavó con agua y jabón, y para la antisepsia se utilizó solución iodada. Para el lavado prepucial se utilizó una mezcla de oxitetraciclina al $5 \%$ en solución salina (ss) al 0,9\% (50 mL/ bolsa de 500 $\mathrm{mL}$ de ss). Para la realización de la cirugía fue necesario un instrumental general.

\section{Descripción de la técnica quirúrgica}

Una vez tranquilizado el paciente y realizadas las maniobras de derribo, se hizo lavado y evaluación detallada de la lesión. Se colocó la mordaza por encima del limbo mucocutáneo para evitar la contracción de la mucosa prepucial una vez incidida. Se realizó una incisión en el limbo mucocutáneo y no en piel para reducir las posibilidades de fimosis cicatrizal. Se ligaron los vasos de gran calibre con puntos de transfixión con material absorbible acidó poliglicólico del calibre 0. Para la hemostasia de los vasos pequeños fue suficiente el pinzamiento y torsión. La divulsión se realiza en $360^{\circ}$ hasta conseguir el desprendimiento de todo el tejido afectado incluyendo la mucosa prepucial dañada. Una vez practicada la correcta hermostasis se procedió sujetar la mucosa con pinzas de goma para evitar la retracción, así mismo se le colocaron 4 puntos de forma cardinal con sutura de nylon monofilico para expandir la mucosa y poder suturarla a la piel, esos mismo puntos de sujeción se fijaron con punto de cirujano y posteriormente se colocaran 4 puntos entre ellos. 


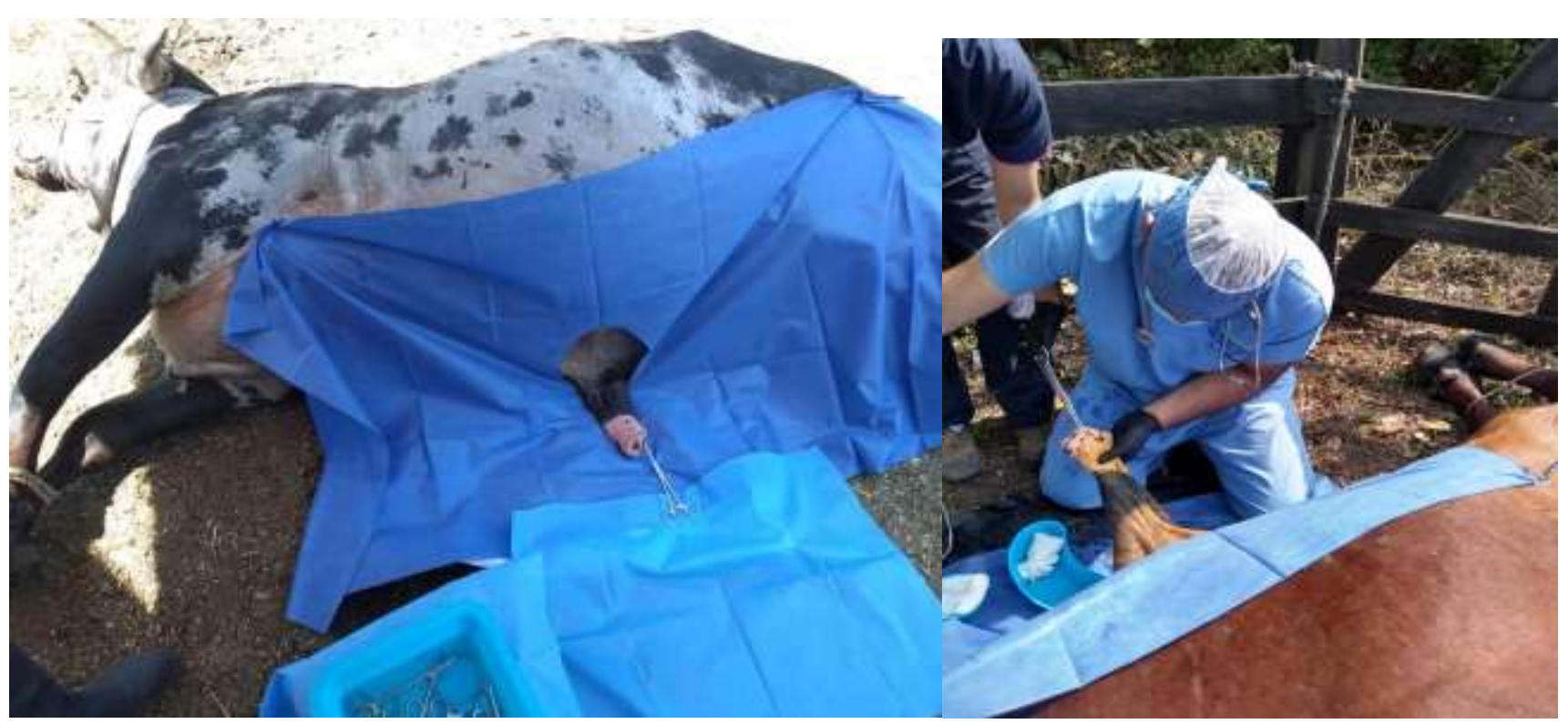

Figura 1. Parte del procedimiento.

\section{Tratamiento}

Se aplicó dexametaxona a $20 \mathrm{mg} / \mathrm{kg}$ p. v. por vía intramuscular una vez concluida la cirugía, y se complementó con enzimas proteolíticas (quimotripsina) por un periodo de cuatro días. Penicilina G procaína a dosis de 20000 UI / kg cada 12 hrs por 5 días, vía intramuscular profunda, además de curaciones locales, durante 7 días.

\section{RESULTADOS Y DISCUSIÓN}

Los 10 toros operados con la técnica descrita tuvieron recuperación en un período de 90 días posoperatorio en promedio, dos de ellos tuvo estenosis cicatrizal. Los 8 toros se recomendaron empezar a trabajarlos como reproductores a los 90 días posoperatorio. Lo resultados del presente estudio coinciden con lo reportado en Colombia por Amaya (1979) (1), quien utilizando la técnica con corte lateral, bilateral en $\mathrm{V}$ y disección de mucosa total de un total de 18 toros operados, todos lograron la recuperación a los 20 días posoperatorio. En este contexto, otro estudio realizado en Colombia por Mastoby et al., (2017) (5), empleando la técnica de postioplastia de 25 toros cebuínos con postitis crónica ulcerativa. La postioplastia es una técnica quirúrgica eficiente porque de los 25 toros intervenidos 22 no tuvieron complicación alguna; retornaron a su vida reproductiva en un lapso comprendido entre dos y tres meses luego de la cirugía. En dos de los toros se presentó miasis complicante atribuida más que todo a un mal manejo posoperatorio. Solo un toro quedó con el ostio prepucial estrecho, posiblemente como resultado de estenosis cicatrizal. Estas técnicas quirúrgicas difieren de la practicada en este estudio al hacer disección total de la mucosa.

\section{CONCLUSIÓN}

Se concluye que la técnica modificada de postioplastia empleada en este estudio tuvo resultados favorables. Se considera que si el posoperatorio no se lleva correctamente trasciende seriamente en el éxito o fracaso de esta cirugía. 


\section{LITERATURA CITADA}

Amaya P.G. 1979. Acrobustitis en toros cebú y Santa Gertrudis -tratamiento quirúrgico. Noticias Médico-Veterinarias. NG. Elwert Universitäts-und Verlagsbuchhandlung Marburg/Lahn. 154-161.

Bicudo SD, Siqueira JB, Meira C. Patologías do sistema reprodutor de touros. Biológico (São Paulo). 2007; 69 (2):43-8.

Deriveaux J. Fisiopatología de la reproducción e inseminación artificial de los animales domésticos. Zaragoza: Acribia, 1967.

Isa JF, Magembe SR, Naude TW. Devil's claw (Harpagophytum procumbens) in a Brahman's preputial sheath: a case report from Botswana. J S Afr Vet Assoc. 2001; 72

(1):55-6. https://doi.org/10.4102/jsava. v72i1.612

Mastoby Miguel Martínez-Martínez, José Alberto Cardona-Álvarez, Diana Marcela Pérez-Berrío. Postioplastia en toros cebuínos del departamento de Córdoba, Colombia. Rev. Med. Vet. ISSN 0122-9354 ISSNe 2389-8526: Bogotá (Colombia) No 35: 35-44, juliodiciembre del 2017 https://doi.org/10.19052/mv.4387

Memom M, Dawson L, Usenik E. Preputial injuries in beef bulls: 172 cases (19801985). J Am Vet Med Assoc. 1988; 193(4):481-5.

Nazario W, Camargo V, Santiago A. Postite oclusiva e fístula prepucial em bovinos traumatizados pela planta Eryfium floribundum (Caraguatá). Atualidades Veterinárias. 1975;26:14-8.

Rabelo R, Silva L, Viu M, Romani A, Alves C, Fernandes J, Castro C. Acrobustite bovina: revisão de literatura. Rev CFMV. 2006; 37:29-36.

Siqueira V, Bernis W, Bernis F. Acropostite bovina: nova técnica cirúrgica e seu tratamento. En: Congresso Brasileiro de Cirurgia e Anestesiologia Veterinária, 2000.

St Jean G. Males reproductive surgery. Vet Clin North Am Food Anim Pract. 1995; 11 (1):55-93. https://doi. org/10.1016/S0749-0720(15)30509-0

Sarma B, Saikia J, Pathak C. Surgical removal of a preputial growth in a bullock. Indian Vet J. 1993; 70 (9):849-50.

Turner S, McIlwrith W. Técnicas cirúrgicas em animais de grande porte. São Paulo: Roca; 1985. 
Copyright (c) 2019 Ronnie de Jesús Arieta Rơn án, B. A Bailón, J. M. E. Graillet, F. J. A. Fernández, G. L. C. Alvarado y M. M. Martinez

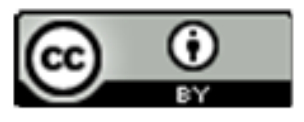

Este tex to está protegido por una licencia licencia Creative Commons 4.0.

Usted es libre para Compartir —copiar y redistribuir el material en cualquier medio o form ato- y Adaptar el documento —remezclar, transformar y crear a partir del material- para cualquier propósito,, incluso para fines comerciales, siempre que cumpla la condición de:

Atribución: Usted debe dar crédito a la obra original de manera adecuada, proporcionar un enlace a la licencia, e in dicar si se han realizado cam bios. Puede hacerlo en cualquier form a razonable, pero no de form a tal que sugiera que tiene el apoyo del licenciante o 10 recibe por el uso que hace de la obra.

Resumendelicencia - Textocompletodelalicencia 